\title{
ILCEA
}

Revue de l'Institut des langues et cultures

d'Europe, Amérique, Afrique, Asie et Australie

$26 \mid 2016$

Mémoire, vérité et justice en Uruguay

\section{La scène de débat autour de l'annulation de la Loi de caducité de la prétention punitive de l'État en Uruguay (2011) : positionnements présidentiels et enjeux politiques}

La escena de debate en torno a la anulación de la Ley de Caducidad de la

Pretensión Punitiva del Estado en Uruguay (2011): posicionamientos

presidenciales y desafíos políticos

The Scene of Debate about the Annulation of the Law on the Expiration of the

Punitive Claims of the State in Uruguay (2011): Presidential Positions and

Political Challenges

\section{Ana Soledad Montero}

\section{(2) OpenEdition}

\section{Journals}

Electronic version

URL: http://journals.openedition.org/ilcea/3923

DOI: 10.4000/ilcea.3923

ISSN: 2101-0609

\section{Publisher}

UGA Éditions/Université Grenoble Alpes

\section{Printed version}

ISBN: 978-2-84310-334-6

ISSN: $1639-6073$

\section{Electronic reference}

Ana Soledad Montero, «La scène de débat autour de l'annulation de la Loi de caducité de la prétention punitive de l'État en Uruguay (2011) : positionnements présidentiels et enjeux politiques », ILCEA [Online], 26 | 2016, Online since 24 June 2016, connection on 20 April 2019. URL : http:// journals.openedition.org/ilcea/3923; DOI : 10.4000/ilcea.3923

This text was automatically generated on 20 April 2019

(c) ILCEA 


\section{La scène de débat autour de l'annulation de la Loi de caducité de la prétention punitive de l'État en Uruguay (2011) : positionnements présidentiels et enjeux politiques}

La escena de debate en torno a la anulación de la Ley de Caducidad de la

Pretensión Punitiva del Estado en Uruguay (2011): posicionamientos

presidenciales y desafíos políticos

The Scene of Debate about the Annulation of the Law on the Expiration of the

Punitive Claims of the State in Uruguay (2011): Presidential Positions and

Political Challenges

Ana Soledad Montero

\section{Introduction}

1 Au cours des dernières années, les pays du Cône Sud ont connu un processus significatif de révision du passé récent, associé à l'avènement dans la région de gouvernements de gauche ou progressistes. Dans les discours présidentiels (Néstor Kirchner et Cristina Fernández de Kirchner en Argentine, Tabaré Vázquez et José Mujica en Uruguay, Michelle Bachelet au Chili, Luis Lula da Silva et Dilma Rousseff au Brésil), ainsi que dans les politiques publiques, dans les initiatives législatives et dans l'atmosphère sociale et culturelle en général, la mémoire des dernières dictatures militaires s'est avérée centrale. En particulier, en Uruguay, l'arrivée au pouvoir de la gauche via le Frente Amplio, avec Tabaré Vázquez en 2005 puis José Mujica en 2010, a ouvert la voie à un nouvel examen d'un passé censé clos. 
2 Cela dit, dans cet article nous nous attachons à analyser les disputes autour d'un événement spécifique: le processus d'annulation de la Loi de caducité de la prétention punitive de l'État en Uruguay, une sorte de loi « d'amnistie » qui empêchait la poursuite des responsables de crimes contre l'humanité perpétrés pendant la dictature militaire. En octobre 2011, le Parlement uruguayen a adopté la loi n 18.831, destinée à suspendre les effets de la Loi de caducité, qui rétablit la prétention punitive de l'État et déclare les crimes commis par la dictature militaire crimes contre l'humanité.

Nous envisageons cet événement comme une "scène litigieuse ", à savoir un espace discursif où prend place, selon les termes de Rancière (1995), un litige ou une controverse politique, et dont l'enjeu n'est autre que la définition de la communauté politique ellemême.

Notre but est de reconstruire les principales quaestio (en termes rhétoriques, l'objet de discussion, le différend qui sépare et en même temps relie les participants d'un débat ${ }^{1}$ ) et de retracer les différentes positions exposées dans chacune des scènes analysées, en dévoilant les positions officielles, et en particulier les discours présidentiels. Dans notre cas d'analyse, l'étude des discours présidentiels semble particulièrement pertinente dans la mesure où l'image de José Mujica, président lors de l'annulation de la loi, était étroitement liée avec la mémoire du passé récent : en effet, il convient de rappeler que J. Mujica, en tant que chef de file de l'organisation politique et armée MLN-Tupamaros, avait participé à quelques épisodes armés et avait été emprisonné pendant treize ans avec d'autres dirigeants tupamaros.

5 De ce point de vue, notre analyse a pour ambition de proposer une réflexion sur la nature litigieuse de la scène de débat sur l'annulation de cette loi «d'amnistie » en Uruguay. Notre étude donnera à voir que les controverses ont touché à des questions substantielles portant sur les fondements de la communauté politique autour d'un «tort » (Rancière, 1995) de nature humanitaire.

6 La mémoire impliquée dans cette scène litigieuse est ainsi un champ de batailles politiques par rapport au passé qui a des effets significatifs sur le présent. L'analyse des disputes concernées par la mise en place d'une mémoire collective nous permettra de mettre en évidence comment se façonnent les récits "officiels» qui informent les identités politiques en Uruguay.

7 Notre corpus d'analyse comprend une série de discours présidentiels, ainsi que des déclarations d'acteurs significatifs du gouvernement et de l'opposition : des ministres, des fonctionnaires, des sénateurs et des députés (dans le cadre des débats parlementaires). Comme on peut le voir, nous n'analysons que des discours politiques dans un sens restreint, c'est-à-dire, des discours prononcés par des fonctionnaires ou des personnalités publiques du gouvernement.

\section{Dictature et démocratie en Uruguay}

8 Il est bien connu que dans les années 1970 les pays du Cône Sud ont connu un processus d'intense radicalisation politique ayant abouti à la mise en place de dictatures militaires fortement répressives orientées à éliminer la "subversion ». La dictature uruguayenne commencée le 27 Juin 1973 s'est présentée dès son début comme une « dictature civique et militaire » : elle a disposé la proscription de certains partis politiques, la dissolution des pouvoirs de l'État, la création d'un Conseil d'État contrôlé par les militaires (qui 
fonctionnait en fait comme l'organe suprême du gouvernement), et elle a mis en œuvre une politique répressive laquelle, avec des méthodes différentes de celles employées en Argentine, a engendré un solde de 300 Uruguayens disparus et des milliers de prisonniers, de torturés et d'exilés (Rico et al., 2009).

9 Après la défaite du plébiscite visant à une réforme constitutionnelle, les forces armées ont organisé et négocié une sortie « ordonnée » du pouvoir, au moyen d'un large accord, le Pacte du Club naval, entre des membres des forces armées, du parti Colorado, de l'Union civique et du Frente Amplio².

\section{La scène de débat sur la Loi de caducité en Uruguay}

Issue des conversations du Club naval et d'intenses négociations entre les différents partis politiques et les militaires, la Loi de caducité de la prétention punitive de l'État (ci-après LC) a été adoptée en décembre 1986 puis validée par un référendum et un plébiscite (en 1989 et 2009 respectivement). Elle établissait la clôture des enquêtes menées par le pouvoir judiciaire et empêchait l'État de punir les militaires et les policiers impliqués dans des crimes ayant eu lieu durant la dictature. En outre, dans son quatrième article, elle déclarait que dans les affaires impliquant des personnes disparues ou des enfants, c'était le pouvoir exécutif qui devrait mener les recherches (Marchesi et al., 2013; Allier Montaño, 2010).

11 Depuis son adoption en 1986, cette loi a connu des hauts et des bas: d'abord, la Cour suprême de justice l'a déclarée constitutionnelle (en 1988) puis inconstitutionnelle (en 2009) dans plusieurs cas spécifiques. D’autre part, plusieurs initiatives de la société civile et/ou du gouvernement ont visé son abrogation ou son annulation. Si depuis son arrivée au pouvoir en 2005 le gouvernement du Frente Amplio a mis en œuvre des mesures à fort impact symbolique, politique et juridique en matière de droits de l'homme (Allier Montaño, 2010 ; Lessa, 2011), J. Mujica était censé avancer sur la LC. Ainsi, malgré la défaite au référendum lancé en 2009, en 2011 le Frente Amplio a présenté un projet interprétatif de la LC permettant de contourner les obstacles pour avancer avec les affaires, projet rejeté à la Chambre des députés en raison de désaccords au sein du parti au pouvoir (tandis que le président J. Mujica lui-même s'est manifesté contre le projet d'interprétation, presque la totalité des législateurs s'est soumise à la discipline partisane et a voté pour). Le 30 Juin, le pouvoir exécutif a décrété la révocation des décisions des gouvernements précédents concernant les enquêtes sur les plaintes de violations des droits de l'homme. En outre, début 2011, un verdict de la Cour interaméricaine des droits de l'homme (CIDH) a déclaré la LC incompatible avec la Convention américaine des droits de l'homme dans l'affaire Gelman.

Ce long chemin a abouti à la promulgation de la loi $\mathrm{n}^{\circ} 18.831$. Après une intense session parlementaire, le 27 octobre 2011 le pouvoir législatif a approuvé une nouvelle proposition de loi (présentée par le Frente Amplio), de quatre articles, déclarant que les crimes commis par l'État pendant la dictature civique et militaire étaient des crimes contre l'humanité, rétablissant de la sorte la " prétention punitive de l'État " pour tous les crimes commis entre 1973 et 1985. La loi interdit, de même, de considérer la période de vigueur de la Loi de caducité pour le calcul des termes de prescription ou de caducité. Ainsi, les effets de la LC ont été neutralisés ${ }^{3}$, et par voie de conséquence, la prescription des crimes commis par la dictature, qui aurait pris effet le $1^{\mathrm{er}}$ novembre 2011, a été évitée. 
La nouvelle loi permettrait d'accomplir la décision de la CIDH et d'entreprendre les procès contre les responsables de crimes contre l'humanité. Or, en 2013 la Cour suprême de justice uruguayenne a déclaré l'inconstitutionnalité des articles 2 et 3 , d'où la possible prescription des causes ouvertes depuis 2011.

Une double urgence a traversé le débat politique uruguayen : d'abord, l'imminence de la prescription des crimes ; puis, la nécessité de s'ajuster au verdict de la CIDH. Urgence qui ressortait des fondements mêmes du projet, dont les arguments s'appuyaient presque exclusivement sur l'adéquation aux règles internationales :

[...] rétablir pleinement la prétention punitive l'État signifie accomplir les règles internationales de protection des droits de l'homme. L'obligation primaire de tous les États est de garantir pleinement les droits de toutes les personnes, d'autant plus qu'il s'agit de violations constituant un affront à la dignité humaine. (Fondements du projet de loi, Commission de Constitution et Législation, Journal de sessions de la Chambre des sénateurs, p. 326)

14 En même temps, la proximité du plébiscite de 2009 et les débats qu'il avait rouverts entre les militants et le grand public au sein du Frente Amplio figuraient en toile de fond. Même si le parti au pouvoir a constitué le principal moteur de l'annulation et que ses votes ont été décisifs pour son approbation, le positionnement du président J. Mujica, à contrecourant des luttes historiques de son parti, de la gauche et des mouvements des droits de l'homme a dû affronter la controverse. D'ailleurs, le président s'est vu accuser - même par ses co-partisans ${ }^{4}$ - d'adopter une attitude «tiède» par rapport au sujet, probablement en raison de son besoin de montrer un visage plutôt «pluraliste » et républicain (Garcé, 2010a) moins associé à son passé de guérilla et à son appartenance au Frente Amplio qu'à un ethos d'homme d'État.

15 Nous dirons que la scène polémique uruguayenne (scène dans laquelle nous incluons les débats ayant eu lieu entre avril et octobre 2011, c'est-à-dire entre la première et la deuxième tentative d'annuler la LC après le référendum de 2009) a tourné autour de trois quaestio : le premier grand axe de débat portait sur l'origine de la LC, à savoir sa sanction et sa légitimation dans les deux référendums. Dérivée de la première, la deuxième quaestio concerne la question juridico-politique de la souveraineté. Troisièmement, la discussion a porté sur le statut de la démocratie et de la république.

\section{Une double origine. Sur l'adoption et la légitimation de la Loi de caducité}

16 L'examen de la LC en Uruguay a impliqué une réinterprétation de son origine, une double origine qui renvoie à la fois au moment de son approbation en 1986 et aux consultations populaires qui l'ont ratifiée en 1989 et 2009.

17 L'évaluation du contexte de promulgation de la LC reconnaissait, en général, le consensus (en tant qu'alliance entre les forces armées et les différentes forces politiques) qui était à sa base. Dans cette mesure, quoique le contenu et les effets de cette loi soient questionnés, les arguments ne portaient pas vraiment sur ses « vices d'origine ». Certes, dans le débat parlementaire les disputes interprétatives autour des conversations du Club naval ont resurgi: alors que certains législateurs estimaient qu'elles avaient été contraintes par les menaces militaires, d'autres considéraient que le pacte avait constitué une "claudication" et une "négociation avec les droits humains", d'autres encore, même critiques de la LC, reconnaissaient l'existence de "nobles raisons » visant à 
«pacifier le pays ». Le sénateur frenteamplista R. Michelini, notamment, à la tête du projet d'annulation, a déclaré dans son allocution:

[...] si à l'époque les arguments donnés étaient directement liés à la pacification de la République, nous sommes maintenant en condition de donner réparation aux victimes et de faire justice. Aujourd'hui la société uruguayenne est pacifiée : il est temps de faire justice. (Sénateur R. Michelini, Frente Amplio, Journal de sessions de la Chambre des sénateurs, p. 507)

La discussion sur la LC a engendré, une fois encore, des révisions et des réinterprétations du rôle des différents acteurs et des partis politiques - notamment le Frente Amplio pendant la transition et la dictature (et même au cours des années précédentes). Les arguments ad hominem ont été à l'avant-garde, avec des accusations croisées touchant d'éventuels accords, alliances, oublis, silences et omissions.

Cependant, lors de la discussion sur la légitimité de la LC, ce n'est pas le contexte de sa sanction qui a causé le plus de controverses, mais les deux référendums y étant favorables: considérées comme le "renouvellement» de ce pacte originaire, les consultations populaires instaurent donc une seconde origine. Dans ce cas, l'interprétation variait selon les positions par rapport à la loi : alors que les partisans de la nullité qualifiaient les consultations populaires de "pièce de monnaie jetée en l'air ", ils remettaient en question les conditions de leur réalisation ou leur procédure et évaluaient le résultat comme un véritable échec politique, les politiciens contraires à l'annulation, quant à eux, considéraient les consultations populaires comme tout à fait légitimes :

On dit qu'en 1989 on a voté avec la peur, qu'il n'y avait pas de garanties, et avec cet argument on a convoqué les citoyens à voter de nouveau, vingt ans plus tard. Les citoyens se sont exprimés exactement de la même façon [...]. L'année du Bicentenaire nous ne pouvons pas dire que le peuple uruguayen vote mal parce qu'il n'a pas été suffisamment courageux en 1989 ni suffisamment illustré en 2009. (Député F. Ricagni, Parti national, Journal de sessions de la Chambre des représentants, p. 93)

Comme on le verra, de ces différentes visions sur la légitimité des référendums découle la quaestio suivante : la souveraineté.

\section{La question de la souveraineté}

En Uruguay il y a eu une intense controverse autour de la question de la compétence pour modifier, voire annuler, une loi démocratique: est-ce une compétence du pouvoir législatif, du pouvoir judiciaire, du pouvoir exécutif, ou du peuple lui-même ? Quelle doit être l'importance des traités internationaux dans le droit national? Il s'agit d'une discussion juridique qui s'est déroulée, pourtant, sur un ton plutôt politique dans la mesure où un concept dense et complexe était en jeu: celui de souveraineté. Souveraineté populaire, d'une part, et souveraineté nationale, de l'autre.

Cette question de la souveraineté est liée à l'histoire politique de l'Uruguay, à sa riche tradition dans l'exercice de la démocratie directe et, par conséquent, aux consultations populaires qui ont approuvé, à deux reprises, la LC : l'événement des plébiscites était donc au centre de la scène litigieuse. En effet, nous avons vu que l'invocation des plébiscites était un argument de premier ordre en faveur de la souveraineté populaire, non sans postuler un dilemme théorique et politique complexe : la majorité a-t-elle toujours raison? À cet égard, Tabaré Vázquez, le président sortant au moment du référendum 
de 2009, avait déclaré que «Dans ces domaines la majorité n’a pas le dernier mot » et avait introduit une considération sur le plébiscite de 1989 : « N’oublions pas les conditions dans lesquelles a eu lieu le plébiscite de 1989. On avait très peur. " (Últimas noticias, 22 mars 2011) En ce sens, les partisans de l'annulation arguaient que les droits de l'homme sont au-dessus de la volonté de la majorité. Le sénateur R. Michelini disait dans une interview à la radio : «La majorité n'a pas le droit de demander aux minorités de s'immoler. L'esclavage et le travail des enfants ne se plébiscitent pas ${ }^{5}$.»

Ceux qui s'opposaient à l'annulation de la LC soutenaient pour leur part que la volonté populaire est souveraine et ne peut donc pas être modifiée par la loi. C'est ainsi que le sénateur Colorado P. Bordaberry a nommé la nouvelle loi, sur son compte Twitter, « la loi de méconnaissance de la volonté du peuple». Les opposants affirmaient que la classe politique ne saurait prétendre que « les gens ont eu tort » et qu'il fallait donc interpréter et réparer les erreurs du peuple.

Quelle a été, à cet égard, la position du président? Dans le discours d'ouverture des sessions parlementaires de 2011, avant la promulgation de la loi, Mujica se positionnait comme le "président de tous les Uruguayens ", réaffirmant ainsi sa volonté d'être exclu de la discussion :

[...] je suis évidemment le Président de tous les Uruguayens, et en tant que tel, j’ai l'obligation incontournable et inviolable d'accepter ce que toute la société, par l'intermédiaire de ses représentants ou par elle-même, décide, à condition que cela s'encadre dans le plus strict respect du pacte le plus sacré, celui qui est reflété dans notre Grande Charte. À elle, comme à la justice, nous devons nous consacrer tous, quelle que soit notre classe sociale ou notre position. Elle doit être scrupuleusement respectée, afin de maintenir ce qui est au-delà de toute douleur, au-delà de toute conviction ou de toute passion, le pacte original et la conviction de pouvoir fonctionner comme une nation. Le fait d'outrager cette obligation signifie un renoncement impardonnable à cette décision prise il y a presque deux siècles. $\left(1^{\text {er }}\right.$ mars 2011, Ouverture des sessions parlementaires)

L'exécutif a refusé de se mêler à cette discussion car je suis Président de la nation, de ceux qui ont voté pour nous et de ceux qui n'ont pas voté pour nous. Nous avons dit dès le début que nous voulions construire, dans la mesure du possible, l'unité nationale. [...] cette discussion ne fait pas de bien à l'unité nationale, et c'est pour cela que nous avons sollicité au parti que le Gouvernement soit expressément exclu de la décision. (El País, 17 avril 2011)

En tant que «Président de la nation, de ceux qui nous ont voté pour nous et de ceux qui n'ont pas voté pour nous » Mujica cherchait et même incarnait «l'unité nationale », et déclarait qu'il soumettrait sa décision, "au-delà de toute conviction ou passion ", aux résolutions de la société «par l'intermédiaire de ses représentants » et aux règles de la justice et de la Constitution. Toutefois, ces instances sont dans ce cas en franche contradiction : c'est précisément dans cet interstice, dans cette tension entre la volonté de la société, les décisions politiques, la justice et la Constitution, que le discours mujiquista forge son ethos.

Dans son audition du 12 mai 2011, quelques jours avant le vote de la loi interprétative, Mujica évoquait publiquement et explicitement la question, tout en introduisant une série de tensions :

- Tension, premièrement, entre la volonté populaire et la représentation politique, c'est-àdire, entre la classe politique (en tant qu'aristocratie ou «politiciens professionnels ») et les citoyens ordinaires en premier lieu :

[...] j'accompagne l'intention d'enfouir l'impunité [...] je l'ai toujours pensé. Mais je suis en désaccord avec la voie choisie, pour deux raisons. On est en train de passer 
outre deux référendums. Là nous nous heurtons à un autre principe, celui du respect de la volonté du peuple. [...] Je n'aime pas que les politiciens professionnels transmettent aux gens que la démocratie est une question de technicismes juridiques [...] qu'ils peuvent tirer des conclusions très différentes du bon sens. [...] Ce n'est pas bon que les grandes règles de la démocratie deviennent des affaires d'aristocratie intellectuelle. Ce n'est pas bon de dire aux gens qu'ils ne comprennent pas lorsqu'ils votent. Ce n'est pas bon de leur dire qu'ils ont été consultés par erreur, car ils n'avaient pas vraiment le droit de décider quoi que ce soit. Je crains que beaucoup ne sentent qu'il y a des pièges dans toute cette affaire. Telle est la raison majeure pour laquelle je suis en désaccord avec le projet en cours de discussion. (Audition du 12 mai 2011)

- Tension, deuxièmement, entre le pouvoir exécutif et le pouvoir législatif :

Le gouvernement, l'exécutif lui-même, ayant une opinion, dans le domaine de la décision va rester en-dehors. [...] Nous devons avoir l'unité. Ce n'est pas que nous manquions d'outils juridiques pour le faire. [...] Ce n'est qu'une signature [...] mais ce n'est pas la voie que le gouvernement suivra. Nous ne le ferons pas parce que nous croyons que le Parlement représente la nation de façon beaucoup plus directe que la présidence et donc sa volonté ne doit pas être obstruée par le président. [...] Tous les partis et tous les secteurs pour lesquels les gens ont voté y sont présents. Toutes les nuances que la carte politique offre sont là dans le Parlement. Et ces nuances ne sont pas dans la Tour exécutive. Là, il n'y a que ceux qui, comme nous, regardent la réalité depuis un angle, le nôtre, inévitablement lié à notre vision. (Audition du 12 mai 2011)

- Tension, finalement, entre les majorités et les minorités :

[...] On est en train d'utiliser une majorité parlementaire minuscule obtenue avec $1 \%$ des votes. [...] La majorité est tout à fait légitime, mais il faut reconnaître qu'elle tient à un fil. Et cela devrait nous obliger à être prudents lorsque nous intervenons dans des affaires de cette dimension, qui peuvent faire l'histoire de ce pays pour les 20 ou 30 prochaines années. Il y a une énorme minorité qui a voté pour d'autres partis. À son tour, à l'intérieur du Front lui-même il y a une autre minorité énorme qui est en désaccord avec le projet de loi. Or l'assemblée de notre Parti va les obliger à voter avec discipline. C'est légitime, c'est légal, c'est-ce que les règles établissent ! Il n'y a pas de doute et nous devons les respecter. Mais permettez-moi de douter que ce soit le mieux pour le pays. (Audition du 12 mai 2011)

Tout en remarquant l'extraordinaire envergure du sujet (une « affaire qui peut influencer l'histoire de ce pays pour les 20 ou 30 années à venir »), le président se situait au-dessus des différences idéologiques, partisanes et politiques, et en exposait les enjeux : d'une part, la question de la volonté populaire, en tant que principe suprême et univoque qui ne devrait pas se voir soumis aux décisions particulières des politiciens, à leurs savoirs techniques ou à leurs intérêts partisans. D'autre part, la question du rôle des pouvoirs de l'État, à savoir le pouvoir législatif en tant que domaine de la diversité, et le pouvoir exécutif en tant que siège de l'unicité, dont les critères de justice sont donc douteux. La question, enfin, des majorités (parlementaires ou populaires) et des "énormes» minorités, qui restituait la question - ouverte, insoluble - de la raison du plus grand nombre et la voix des moins nombreux. En d'autres termes : la majorité a raison car elle exprime la souveraineté populaire, mais les minorités ont, elles aussi, leurs raisons, le pouvoir exécutif n'ayant pas de compétence pour régler cette affaire.

Enfin, on doit remarquer que dans son discours Mujica se montre également comme une figure complexe : il distingue son rôle de président de celui de militant politique, tout en reconnaissant l'impossibilité de dissocier les deux rôles lorsqu'il doit "exprimer son opinion " à ses co-partisans («Il est impossible de scinder le militant politique du Président de la République. Nous sommes très conscients que ces distinctions sont des 
fantaisies »); il scinde également son opinion personnelle de la décision politique à adopter (« Le gouvernement, l'Exécutif en tant que tel, ayant une opinion, dans le domaine de la décision va se maintenir écarté ») tout en affirmant qu'il n'obstruerait pas la volonté parlementaire.

Mais la question de la souveraineté a aussi un ancrage territorial. À plusieurs reprises, la scène de débat uruguayenne a mis l'accent sur le conflit entre les normes nationales et le jus cogens ou l'ordre juridique international. D'un côté, les partisans de l'annulation de la LC réfutaient le concept (qualifié d'«anachronique») de souveraineté nationale et valorisaient l'universalité des droits de l'homme, affirmés dans la législation internationale. D'un autre côté, ceux qui étaient pour la continuité de la LC s'appuyaient sur une notion de souveraineté liée à la Constitution nationale. La Constitution apparaissait alors comme le garant ultime de l'ordre, d'où l'affirmation que l'adéquation constitutionnelle aux traités internationaux conduirait à un « révisionnisme total et absolu", selon les mots d'un sénateur du Parti national. Plus radical était le positionnement de ceux qui remettaient en cause les organisations internationales des droits de l'homme, qualifiées d'« impérialistes » et étrangères à l'Amérique latine. De ce point de vue, les droits de l'homme sont représentés comme « une invention des ÉtatsUnis des années 1970 $»$ (Buriano, 2011 : 197) :

Ces organismes internationaux sont généralement très courageux vis-à-vis des petits pays, et très dociles vis-à-vis des grands, devant lesquels ils s'agenouillent. [... ] [Cette Cour] demande à l'Uruguay ce qu'elle n'ose pas demander aux pays puissants. Donc, ni droits de l'homme, ni tribunaux internationaux, parce qu'on ne peut pas défendre les droits de l'homme en violant la Constitution de la République. (Député J. García, Parti national, Journal de sessions de la Chambre des représentants, p. 68).

Quant à la position du président, il introduit à cet égard une nouvelle tension entre la Constitution et le droit international. Dans une interview Mujica a signalé que le verdict de la CIDH ouvrait un dilemme qui n'était pas « simple » à résoudre dans la mesure où il remettait en cause la décision souveraine du peuple :

En effet, nous sommes condamnés du point de vue international pour avoir maintenu la Loi [de caducité]. Ce n'est pas un problème simple. Il y a des aspects à considérer dans les deux points de vue. C'est un dilemme entre les décisions de notre peuple et la décision du Parlement, qui apparaît comme modifiant le résultat des plébiscites. (El País, 17 avril 2011)

On peut voir que Mujica ne propose aucune "solution " à ces dilemmes : au contraire, il se soustrait du débat tout en exposant les complexités de la décision ${ }^{7}$.

\section{Politique, démocratie, république}

La scène de débat sur l'annulation de la LC a été maintes fois caractérisée comme une scène plus « politique » que juridique : en effet certains ont argumenté que seuls les juges pouvaient réviser les lois, sous peine d' « antéposer les intérêts politiques »; d'autres, plus modérés, affirmaient que les juges pouvaient adapter la législation aux conventions internationales, et qu'il n'était donc pas nécessaire d'adopter une décision politique ; les partisans de l'annulation, quant à eux, jugeaient qu'il fallait fournir aux victimes et aux familles des garanties supplémentaires. c'était la démocratie républicaine. Pour les détracteurs de l'annulation, le système 
républicain était en danger en raison de l'ignorance de la Constitution, qui consacre la primauté de la volonté citoyenne :

« Nous ne sommes pas face à une affaire juridique ou philosophique; ce n'est pas du principe de la loi ou de la justice dont nous parlons ici. Nous sommes confrontés à un problème politique. Ce n'est pas la loi que nous sommes en train de remettre en question, mais le système républicain. [...] C'est la volonté citoyenne qu'on est en train de corriger encore une fois aujourd'hui », prônait le député F. Ricagni du Parti national (Journal de sessions de la Chambre des représentants, p. 93).

Sauvegarder la République impliquait donc de préserver la démocratie : « Nous défendons la démocratie, nous défendons la République. Voilà pourquoi nous ne voterons pas ce projet de loi et pourquoi nous dénonçons cette attaque à la Constitution", soutenait quant à elle la députée Piñeyrúa (Journal de sessions de la Chambre des représentants, p. 99).

Or, les défenseurs de l'annulation de la LC plaidaient eux aussi pour la démocratie et la République : une démocratie - selon le député frenteamplista L. Puig - aussi substantielle que formelle, de sorte que les normes internationales lui imposaient une limite. Par conséquent, la nouvelle loi, loin d'ignorer le mandat citoyen était « une contribution substantielle " à la démocratie. Une démocratie républicaine qui devait encore être renforcée par la pleine reconnaissance du pouvoir judiciaire, obturée par le quatrième article de la LC, qui avait laissé la "Justice aveugle», selon les termes du député Julio Bango, du Frente Amplio ${ }^{8}$. Selon les mots du député frenteamplista Victor Semproni :

Ce qui compte aujourd'hui, à mon avis, c'est de préciser que cette question ne doit pas être résolue sur la base de l'inconstitutionnalité ou la constitutionnalité de ses points. Il s'agit d'une question éminemment politique. Les deux positions sont en contradiction. Il y en a qui croient que la Justice doit agir pleinement, en émettant des décisions et en sanctionnant, et il y en a qui, sur la base d'une loi qui semble inamovible - comme si les lois étaient éternelles et qu'elles ne pouvaient pas être supprimées ou modifiées -, soutiennent que la loi doit rester en place et que rien ne peut la changer. Ainsi, ils défendent ceux qui ont torturé, ceux qui ont violé, ceux qui ont fait disparaitre et qui ont tué des personnes. (Député V. Semproni, Frente Amplio, Journal de sessions de la Chambre des représentants, p. 96)

La décision d'abolir "définitivement l'impunité » après 26 ans était, selon de nombreux législateurs, éminemment politique dans la mesure où elle devait être fondée sur des convictions profondes. Or cela supposait l'existence de «deux positions» indécidables, incommensurables, inconciliables :

«Pour résoudre ce problème que nous avons comme nation aujourd'hui, il n'y a aucune voie, absolument aucune, qui ne puisse être contestée dans son contenu ou dans sa proposition. Je ne vais donc pas entrer dans le débat en espérant convaincre ceux qui ne voteront pas ce projet ", a déclaré Semproni.

Il fallait assumer l'incertitude ouverte par le litige en cours, c'est-à-dire, sa nature politique et radicalement démocratique. C'est en ce sens que Mujica signalait, lors de son audition du 12 avril 2011, que «le climat politique est raréfié. Mais non à cause du manque d'oxygène, au contraire : il y a beaucoup de démocratie dont nous sommes fiers, il y a de l'oxygène en trop, et, c'est connu, cela produit parfois trop d'excitation ». « Il y a beaucoup de démocratie » en Uruguay, et l'excitation démocratique, loin de guider une lecture univoque, ravivait le débat, exposait les positions et les différends, sans jamais vouloir les fermer. Mais quel type de nœud se trame-t-il alors entre politique, république et démocratie? 


\section{Réflexions finales} questions de fond qui concernent la conformation de la communauté politique, ses exclusions, ses inclusions, ses solidarités et ses frontières. Suivant la tradition rhétorique nous avons dit qu'en Uruguay ces scènes de débat étaient structurées autour de quelques quaestios, clivages ou nœuds polémiques qui touchent une question fondamentale: que faire avec le passé de violence et de crime lorsqu'il s'agit d'établir ou de consolider une communauté démocratique?

disposition de la scène litigieuse de mémoire déployée autour de l'annulation/ neutralisation de la Loi de caducité présentait des traits particuliers: tout d'abord, l'événement législatif était inscrit dans une longue histoire de débats, d'initiatives, de frustrations et de réflexions au sein du parti au pouvoir, dans les organisations de droits de l'homme et dans la société civile elle-même, débats qui après une tentative ratée et avec une «maigre » majorité parlementaire, ont abouti à la promulgation de la loi $\mathrm{n}^{\circ}$ 18.831 de restitution de la capacité punitive de l'État. En effet, la société uruguayenne s'était donné elle-même l'occasion, pendant plus de vingt ans, de discuter ces questions dans un cadre institutionnel et réglé: les assemblées partisanes, les consultations populaires, le Parlement, les tribunaux. Maintes fois répétées, elles étaient des expériences de mise en commun d'arguments et de contre-arguments, dont la scène de 2011 n'est qu'une de plus. C'est pourquoi le projet de restitution du pouvoir punitif de l'État n'a pas été perçu comme strictement lié à la figure du président, même pas au Frente Amplio, et encore moins comme un événement absolument exceptionnel. S'il est vrai que la nouvelle législation ouvrirait la voie de la justice et de la vérité exigée par de larges secteurs de la société, il est également vrai que depuis le mandat de Vázquez (2005-2010) quelques procès avaient déjà été entamés (Allier Montaño, 2010).

36 Le discours de Mujica ne propose pas un ton de refondation par rapport au passé ; au contraire, il réclame un dépassement et une distance critique vis-à-vis des conflits du passé. Quant à la LC, sa double origine - son adoption en 1986, mais surtout les deux consultations populaires qui ont renouvelé ce pacte originaire - est un prisme qui rend visible les tensions que le débat comporte : tension entre le principe de la souveraineté populaire et celui de la Constitution, entre la volonté populaire et les décisions de ses représentants, entre les décisions exécutives et le rôle du pouvoir législatif, entre les majorités et les minorités, entre les dispositions internationales et le principe de la souveraineté nationale, entre la justice punitive et la recherche de la vérité, entre la république et la démocratie.

Certes Mujica a bien souvent exprimé son «opinion personnelle», mais non sans la distinguer de la décision politique à prendre, et non sans en remarquer les tensions et paradoxes, des tensions insolubles, indécidables, impossibles à clore par une disposition ou une décision. Des tensions indiquant la précarité du politique, l'instabilité et la fragilité de toute intervention sur ce qui est de l'ordre du commun. Chez Mujica la mémoire du passé apparaît comme une interrogation qui suscite la discussion et qui reste ouverte, inachevée, une question qui fait face, comme l'a suggéré le président lui-même, à des «blessures tellement profondes qu'elles ne vont peut-être jamais se refermer » et avec lesquelles « il faudra apprendre à vivre » ( $1^{\mathrm{er}}$ mars 2011).

ILCEA, 26 | 2016 


\section{BIBLIOGRAPHY}

ABRAHAM Nicolás \& MATTEI Eugenia (2012), « Nuevo comienzo democrático en Uruguay: la Ley de Caducidad en querella », African Yearbook of Rhetoric, 3(2).

ALLIER MONTAÑo Eugenia (2010), Batallas por la memoria. Los usos políticos del pasado reciente en Uruguay, Montevideo : Trilce.

BURIANo Ana (2011), « Ley de Caducidad en Uruguay y esencia ético-política de la izquierda », Perfiles Latinoamericanos, 38.

GARCÉ Adolfo (2010a), « Uruguay 2009: de Tabaré Vázquez a José Mujica », Revista de Ciencia Política , 30(2), 499-535.

GARCÉ Adolfo (2010b), « De guerrilleros a gobernantes: el proceso de adaptación del MLNTupamaros a la legalidad y a la competencia electoral en Uruguay (1985-2009) », dans E. Rey Tristán \& P. Calvo González, XIV Encuentro de Latinoamericanistas Españoles (congreso internacional, sept. 2010, Santiago de Compostela, España, Universidade de Santiago de Compostela, Centro Interdisciplinario de Estudios Americanistas Gumersindo Busto), 1598-1619, < http://halshs.archives-ouvertes.fr/docs/00/53/12/45/PDF/AT11_Garce.pdf> (consulté le 8 novembre 2013).

LESSA Francesca (2011), « Las múltiples máscaras de la impunidad: la Ley de Caducidad, desde el Sí Rosado hasta los desarrollos recientes », dans G. Fried \& F. Lessa, Luchas contra la impunidad. Uruguay 1985-2011, Montevideo : Trilce.

MARChESI Aldo, BARDAZANo Gianella, Giorgi Álvaro DE, GIORgI Ana Laura DE, SEMPol Diego, Duffau Nicolás \& LUJÁN Diego (2013), La Ley de Caducidad, un tema inconcluso. Momentos, actores y argumentos (1986-2013), Montevideo : Trilce.

MEYER Michel (2004), La rhétorique, Paris : Presses universitaires de France.

MONTERo Ana Soledad (2013), « Memoria, militancia y refundación política. Los discursos oficiales sobre el pasado reciente en Argentina (2003-2007) y Uruguay (2010-2012) », dans V Congreso de la Sociedad Argentina de Análisis Político (SAAP), Paraná, Argentina.

Plantin Christian (2005), L'argumentation. Histoire, théories et perspectives, Paris : Presses universitaires de France.

RANCIÈrE Jacques (1995), La mésentente, Paris : Galilée.

Rico Álvaro, Demasi Carlos, MARCHESI Aldo, MARKARIAN Vania \& YAFFÉ Jaime (2009), Uruguay 1973-1985. La dictadura cívico-militar, Montevideo : Ediciones de la Banda Oriental.

\section{Sources journalistiques}

La Nación, 19 septembre 2009, " No sé qué ideología tienen los Kirchner », < www.lanacion.com.ar/1173730-no-se-que-ideologia-tienen-los-kirchner> (consulté le 15 octobre 2014). 
Télam [s.d.] : « Luis Wilfredo Puig: "Pepe Mujica tiene una deuda pendiente: hacer algo por los derechos humanos" ", <http://memoria.telam.com.ar/noticia/dd-hh--el-diputado-puig-critico-apepe-mujica_n2794> (consulté le 15 octobre 2014).

Ultimas noticias, 22 mars 2011, « Vázquez se mostró partidario de eliminar la Ley de Caducidad », < www.ultimasnoticias.com.uy/Edicion-UN/articulos/prints-2011mar22/act05.html> (consulté le 15 octobre 2014).

El País, 17 avril 2011, "Yo no miro atrás, pero no puedo imponer a los ciudadanos mi manera de ser ", <http://elpais.com/diario/2011/04/17/domingo/1303012356_850215.html> (consulté le 15 juin 2016).

\section{Sources documentaires}

RÉPUBLIQUE ORIENTALE DE L'URUGUAy, Journal de sessions de la Chambre des sénateurs, tome $488, \mathrm{n}^{\circ} 115$, 25 octobre 2011.

RÉPUBLIQUE ORIENTALE DE L'URUGUAY, Journal de sessions de la Chambre des représentants, $\mathrm{n}^{\circ} 3750$, 26 octobre 2011.

MUjICA José, Discours présidentiels et auditions radiales, téléchargés à partir du site < www.presidencia.gub.uy> (consulté en mai 2014).

\section{NOTES}

1. Meyer souligne que « s'il n'y avait pas un problème, une question [...] il n'y aurait pas de débat, même pas de discussion» (2004, p. 10). Selon Plantin (2005, p. 59) les «questions argumentatives » sont les thèmes qui organisent un conflit discursif et qui structurent l'échange argumentatif. Nous utilisons le terme latin quaestio pour le distinguer des mots - liés mais, nous le verrons, différents - question et problème.

2. Le Frente Amplio est un parti fondé en 1971 réunissant divers secteurs de la gauche ; le Partido Nacional et le Partido Colorado - partis multi-idéologiques à orientation libérale et conservatrice, respectivement - sont les partis traditionnels en Uruguay.

3. Il existe un point de débat juridique sur le fait de savoir si le rétablissement de la capacité punitive de l'État suppose l'annulation, l'abrogation (tacite ou explicite) ou bien la suspension de la LC. Abraham et Mattei (2012) préfèrent le terme de «neutralisation » des effets de la LC, au sens schmittien du terme.

4. Dans une interview en Argentine, le législateur frenteamplista L. W. Puig, par exemple, affirmait que Mujica est un « compagnon honnête et fidèle » mais qu'il a une « dette » vis-à-vis des droits de l'homme, et qu'il donne des messages contradictoires reproduisant la théorie des deux démons (Télam, s/d).

5. Interview à la Radio Nacional (Argentine) le 19 mai 2011 : < www.youtube.com/watch? v=FhJ_ny-IaZY>.

6. Ce geste peut être interprété comme l'écho d'un trait idéologique tupamaro: l'indépendance des critères face à toute forme de colonisation (même la soviétique), et son empreinte nationaliste (Garcé, 2010b : 1602).

7. Dans des anciennes déclarations, énoncées sans l'investiture présidentielle, Mujica avait signalé le trait « impérialiste » des organismes internationaux. Dans une interview télévisée dans les années 1990 en Espagne, Mujica reconnaissait, à l'égard de l'emprisonnement de Pinochet en Angleterre et de son procès en Espagne, qu'il était « heureux et triste » à la fois : heureux parce que Pinochet serait finalement jugé, et triste parce qu'il avait « du mal à croire aux tribunaux 
internationaux ». Allier Montaño (2010: 228) signale que la société uruguayenne s'est divisée entre ceux qui appuyaient le procès de Pinochet et ceux qui croyaient qu'il ne devait pas avoir lieu, ou qu'il devait avoir lieu au Chili.

8. Mais cette Justice, qui devait être encouragée et à laquelle, selon Mujica, la décision présidentielle devait se soumettre, était elle-même remise en cause par le président. Bien que jamais depuis une position d'énonciation présidentielle, Mujica a signalé maintes fois une tension entre le principe de justice (rétributive), la demande de "vérité » et le principe humanitaire. En fait, Mujica avait le «projet, frustré en raison de l'opposition de la plupart des membres du FA, d'encourager la libération - pour des raisons humanitaires - des prisonniers âgés de plus de 70 ans accusés de violations de droits de l'homme » (Garcé, 2010b : 533) en échange d'information véridique, suivant sur certains aspects le modèle sud-africain. Dans une interview de 2009, avant les élections présidentielles, Mujica établissait en effet un parallèle entre justice et vengeance : «Je veux connaître la vérité, mais la justice, je m'en fous. [...] Au niveau personnel, moi j’ai pensé : “S’ils disent la vérité, je leur commute la peine." Si c'est la vérité qui m’intéresse ! Mais les sociétés peuvent-elles tolérer ça? Parce que la Justice pue la vengeance.» (La Nación, 19 septembre 2009)

\section{ABSTRACTS}

In this paper we analyze the polemical disputes about the annulation of the Law on the Expiration of the Punitive Claims of the State in Uruguay, law that prevented the prosecution of those responsible for crimes against humanity during the military dictatorship and that was cancelled in 2011. The main goal of this paper is to reconstruct the different positions deployed in the political, parlamentary and media debate, with emphasis on official positions, and specially on presidential speeches.

Dans cet article nous nous attachons à analyser les disputes autour du processus d'annulation de la Loi de caducité de la prétention punitive de l'État en Uruguay, une loi empêchant le jugement des responsables des crimes contre l'humanité pendant la dernière dictature militaire qui a été annulée en 2011. Notre contribution vise à examiner les positionnements discursifs des intervenants dans le débat (parlementaire, politique et médiatique), en mettant spécialement l'accent sur les discours présidentiels dans leur rapport avec le passé récent. Par ce biais, nous avons pour ambition de contribuer à la réflexion sur les enjeux de tout processus d'élaboration d'un passé traumatique visant à la consolidation d'une communauté démocratique.

En este artículo nos proponemos abordar las disputas en torno al proceso de anulación de la Ley de Caducidad de la Pretensión Punitiva del Estado en Uruguay, ley que impedía el juzgamiento de los responsables de delitos de lesa humanidad durante la última dictadura militar y que fue anulada en el año 2011. Nuestro objetivo es reconstruirlos posicionamientos discursivos de los partícipes del debate (parlamentario, político y mediático), poniendo especialmente el acento en los discursos presidenciales en su vínculo con el pasado reciente. De ese modo, nos proponemos contribuir a la reflexión sobre los principales desafíos de todo proceso de elaboración de un pasado traumático en vistas a la consolidación de una comunidad democrática. 
INDEX

Keywords: Law on the Expiration on the Punitive Claims, memory, political discourse, Uruguay Mots-clés: Loi de caducité, mémoire, discours politique, Uruguay

Palabras claves: Ley de Caducidad, memoria, discurso político, Uruguay

\section{AUTHOR}

\section{ANA SOLEDAD MONTERO}

Universidad de Buenos Aires (UBA), Consejo Nacional de Investigaciones Científicas y Técnicas (CONICET), Argentina.

Ana Soledad Montero est sociologue et docteure en philosophie et lettres (UBA, Argentine). Elle est actuellement chercheuse au Conseil national de la recherche scientifique et technique (CONICET) en Argentine, ainsi que professeure à l'Université de Buenos Aires (UBA) et l'Université nationale de San Martín. Ses recherches se situent au carrefour de différentes disciplines telles que l'analyse du discours politique, les études sur la mémoire et la théorie politique. Elle est l'auteure du livre «iY al final un día volvimos!». Los usos de la memoria en el discurso kirchnerista (Buenos Aires, Prometeo, 2012) et de plusieurs articles scientifiques. 